\title{
Photonic crystals for the application to spectrometers and wavelength filters
}

\author{
Yasuo Ohtera ${ }^{\text {a) }}$ and Hirohito Yamada \\ Graduate School of Engineering, Tohoku University, Aramaki-Aza-Aoba 6-6-05, \\ Sendai, Miyagi, 980-8579 Japan \\ a) ohtera@ecei.tohoku.ac.jp
}

\begin{abstract}
This paper reviews the application of photonic crystals $(\mathrm{PhCs})$ and related periodic structures to spectrometers and wavelength filters. In the first half of the paper in-plane type spectrometers utilizing superprism effect are reviewed. In the latter half out-of-plane and surface-normal type devices, where guided mode resonance (GMR) plays a center role, are described. Finally, Bragg reflection type wavelength filters are dealt with.
\end{abstract}

Keywords: spectrometer, photonic crystal, superprism, subwavelengh grating, guided mode resonance, wavelength filter

Classification: Fiber optics, Microwave photonics, Optical interconnection, Photonic signal processing, Photonic integration and systems

\section{References}

[1] J. D. Joannopoulos, S. G. Johnson, J. N. Winn, and R. D. Meade, Photonic Crystals, 2nd ed., Princeton University Press, 2008.

[2] E. Yablonovitch, "Photonic band-gap structures," J. Opt. Soc. Am. B, vol. 10, no. 2, pp. 283-295, 1993.

[3] C. P. Bacon, Y. Mattley, and R. DeFrece, "Miniature spectroscopic instrumentation: Applications to biology and chemistry," Rev. Sci. Instr., vol. 75 , no. 1 , pp. 1-16, 2004.

[4] R. F. Wolffenbuttel, "State-of-the-Art in integrated optical microspectrometers," IEEE Trans. Instrum. Meas., vol. 53, no. 1, pp. 197-202, 2004.

[5] D. Sander and J. Muller, "Selffocusing phase transmission grating for an integrated optical microspectrometer," Sensors and Actuators A, vol. 88, pp. 1-9, 2001.

[6] J.-J. He, B. Lamontagne, A. Delage, L. Erickson, M. Davies, and E. S. Koteles, "Monolithic integrated wavelength demultiplexer based on a waveguide Rowland circle circle grating in InGaAsP/InP," J. Lightwave Technol., vol. 16, no. 4, pp. 631-638, 1998.

[7] J. Song and N. Zhu, "Design and fabrication of compact etched diffraction grating demultiplexers based on a-Si nanowire technology," Electron. Lett., vol. 44, no. 13, pp. 816-817, 2008.

[8] B. Momeni, M. Askari, E. S. Hosseini, A. Atabaki, and A. Adibi, "An on-chip silicon grating spectrometer using a photonic crystal reflector," J. Opt., vol. 12, 035501, 2010. 
[9] E. Chow, A. Grot, L. W. Mirkarimi, M. Sigalas, and G. Girolami, "Ultracompact biochemical sensor built with two-dimensional photonic crystal microcavity," Opt. Lett., vol. 29, no. 10, pp. 1093-1095, 2004.

[10] P. St. J. Russell, T. A. Birks, and F. D. Lloyd-Lucas, "Photonic Bloch waves and photonic band gaps," Confined Electrons and Photons, Eds. E. Burstein and C. Weisbuch, pp. 585-633, Plenum Press, New York, 1995.

[11] H. Kosaka, T. Kawashima, A. Tomita, M. Notomi, T. Tamamura, T. Sato, and S. Kawakami, "Superprism phenomena in photonic crystals," Phys. Rev. B, vol. 58, no. 16, pp. R10096-R10099, 1998.

[12] H. Kosaka, T. Kawashima, A. Tomita, M. Notomi, T. Tamamura, T. Sato, and S. Kawakami, "Self-collimating phenomena in photonic crystals," Appl. Phys. Lett., vol. 74, no. 9, pp. 1212-1214, 1999.

[13] T. Kawashima, K. Miura, T. Sato, and S. Kawakami, "Self-healing effects in the fabrication process of photonic crystals," Appl. Phys. Lett., vol 77, no. 16 , pp. 2613-2615, 2000.

[14] L. Wu, M. Mazilu, T. Karle, and T. F. Krauss, "Superprism phenomena in planar photonic crystals," IEEE J. Quantum Electron., vol. 38, no. 7, pp. 915-918, 2002.

[15] M. Notomi, "Theory of light propagation in strongly modulated photonic crystals: Refractionlike behavior in the vicinity of the photonic band gap," Phys. Rev. B, vol. 62, no. 16, pp. 10696-10705, 2000.

[16] T. Baba, T. Asatsuma, and T. Matsumoto, "Negative refraction in photonic crystals," MRS Bulletin, vol. 33, pp. 927-930, 2008.

[17] B. Momeni, J. Huang, M. Soltani, M. Askari, S. Mohammadi, M. Rakhshandehroo, and A. Adibi, "Compact wavelength demultiplexing using focusing negative index photonic crystal superprisms," Opt. Express, vol. 14, no. 6, pp. 2413-2422, 2006.

[18] T. Matsumoto, T. Asatsuma, and T. Baba, "Experimental demonstration of a wavelength demultiplexer based on negative-refractive photoniccrystal components," Appl. Phys. Lett., vol. 91, no. 9, 091117, 2007.

[19] A. Bakhtazad and A. G. Kirk, "1-D slab photonic crystal k-vector superprism demultiplexer: analysis, and design," Opt. Express, vol. 13, no. 14, pp. 5472-5482, 2005.

[20] T. Matsumoto, S. Fujita, and T. Baba, "Wavelength demultiplexer consisting of Photonic crystal superprism and superlens," Opt. Express, vol. 13, no. 26, pp. 10768-10776, 2005.

[21] A. Khorshidahmad and A. G. Kirk, "Composite superprism photonic crystal demultiplexer: analysis and design," Opt. Express, vol. 18, no. 19, pp. 20518-20528, 2010.

[22] D. Bernier, X. L. Roux, A. Lupu, D. Marris-Morini, L. Vivien, and E. Cassan, "Compact, low cross-talk CWDM demultiplexer using photonic crystal superprism," Opt. Express, vol. 16, no. 22, pp. 17209-17214, 2008.

[23] B. Momeni, E. S. Hosseini, and A. Adibi, "Planar photonic crystal microspectrometers in silicon-nitride for the visible range," Opt. Express, vol. 17, no. 19, pp. 17060-17069, 2009.

[24] E. Cassan and M. Casale, "Dispersion-accumulated diffractioncompensated superprism with two cascaded photonic crystals," J. Opt. Soc. Am. B, vol. 29, no. 6, pp. 1172-1178, 2012.

[25] S.-H. Kim, H. S. Park, J. H. Choi, J. W. Shim, and S.-M. Yang, "Integration of colloidal photonic crystals toward miniaturized spectrometers," Adv. Mater., vol. 22, pp. 946-950, 2010.

[26] M. Neviere, R. Petit, and M. Cadilhac, "About the theory of optical grating coupler-waveguide systems," Opt. Commun., vol. 8, no. 2, pp. 113- 
$117,1973$.

[27] F. V. Laere, G. Roelkens, M. Ayre, J. Schrauwen, D. Taillaert, D. V. Thourhout, T. F. Krauss, and R. Baets, "Compact and highly efficient grating couplers between optical fiber and nanophotonic waveguides," $J$. Lightwave Technol., vol. 25, no. 1, pp. 151-156, 2007.

[28] H. Yamada, M. Nozawa, M. Kinoshita, and K. Ohashi, "Verticalcoupling optical interface for on-chip optical interconnection," Opt. Express, vol. 19, no. 2, pp. 698-703, 2011.

[29] K. A. Bates, L. Li, R. L. Roncone, and J. J. Burke, "Gaussian beams from variable groove depth grating couplers in planar waveguides," Appl. Opt., vol. 32, no. 12, pp. 2112-2116, 1993.

[30] K. Kintaka, J. Nishii, Y. Imaoka, J. Ohmori, S. Ura, R. Satoh, and H. Nishihara, "A guided-mode-selective focusing grating coupler," IEEE Photon. Technol. Lett., vol. 16, no. 2, pp. 512-514, 2004.

[31] D. S. Goldman, P. L. White, and N. C. Anheier, "Miniaturized spectrometer employing planar waveguides and grating couplers for chemical analysis," Appl. Opt., vol. 29, no. 31, pp. 4583-4589, 1990.

[32] E. Hallynck and P. Bienstman, "Photonic crystal biosensor based on angular spectrum analysis," Opt. Express, vol. 18, no. 17, pp. 1816418170, 2010.

[33] N. K. Pervez, W. Cheng, Z. Jia, M. P. Cox, H. M. Edres, and I. Kymissis, "Photonic crystal spectrometer," Opt. Express, vol. 18, no. 8, pp. 82778285, 2010.

[34] S.-H. Kim, S.-K. Kim, and Y.-H. Lee, "Vertical beaming of wavelengthscale photonic crystal resonators," Phys. Rev. B, vol. 73, no. 23, 235117, 2006.

[35] X. Gan, N. Pervez, I. Kymissis, F. Hatami, and D. Englund, "A highresolution spectrometer based on a compact planar two dimensional photonic crystal cavity array," Appl. Phys. Lett., vol. 100, no. 23, 231104, 2012.

[36] Y. Ding and R. Magnusson, "Resonant leaky-mode spectral-band engineering and device applications," Opt. Express, vol. 12, no. 23, pp. 56615674, 2004.

[37] D. Rosenblatt, A. Sharon, and A. A. Friesem, "Resonant grating waveguide structures," IEEE J. Quantum Electron., vol. 33, no. 11, pp. 20382059, 1997.

[38] S. Fan and J. D. Joannopoulos, "Analysis of guided resonances in photonic crystal slabs," Phys. Rev. B, vol. 65, no. 23, p. 235112, 2002.

[39] Z. S. Liu, S. Tibuleac, D. Shin, P. P. Young, and R. Magnusson, "Highefficiency guided-mode resonance filter," Opt. Lett., vol. 23, no. 19, pp. 1556-1558, 1998.

[40] C. J. Chang-Hasnain and W. Yang, "High-contrast gratings for integrated optoelectronics," Adv. Opt. Photon., vol. 4, pp. 379-440, 2012.

[41] J. H. Schmid, W. Sinclair, J. Garcia, S. Janz, J. Lapointe, D. Poitras, Y. Li, T. Mischki, G. Lopinski, P. Cheben, A. Delage, A. Densmore, P. Waldron, and D.-X. Xu, "Silicon-on-insulator guided mode resonant grating for evanescent field molecular sensing," Opt. Express, vol. 17, no. 20, pp. 18371-18380, 2009.

[42] P. C. Mathias, N. Ganesh, L. L. Chan, and B. T. Cunningham, "Combined enhanced fluorescence and label-free biomolecular detection with a photonic crystal surface," Appl. Opt., vol. 46, no. 12, pp. 2351-2360, 2007.

[43] I. D. Block, M. Pineda, C. J. Choi, and B. T. Cunningham, "High sensitivity plastic-substrate photonic crystal biosensor," IEEE Sensors J., 
vol. 8, no. 9, pp. 1546-1547, 2008.

[44] B. T. Cunningham, "Photonic crystal surfaces as a general purpose platform for label-free and fluorescent assays," J. Assoc. Lab. Automation, vol. 15, no. 2, pp. 120-135, 2010.

[45] B. T. Cunningham, P. Li, S. Schulz, B. Lin, C. Baird, J. Gerstenmaier, C. Genick, F. Wang, E. Fine, and L. Laing, "Label-free assays on the BIND system," J. Biomolecular Screening, vol. 9, no. 6, pp. 481-490, 2004.

[46] B. T. Cunningham and L. Laing, "Microplate-based, label-free detection of biomolecular interactions: applications in proteomics," Expert Rev. Proteomics, vol. 3, no. 3, pp. 271-281, 2006.

[47] R. Magnusson and M. Shokooh-Saremi, "Widely tunable guided-mode resonance nanoelectromechanical RGB pixels," Opt. Express, vol. 15, no. 17, pp. 10903-10910, 2007.

[48] Q. Wang, D. Zhang, B. Xu, Y. Huang, C. Tao, C. Wang, B. Li, Z. Ni, and S. Zhuang, "Colored image produced with guided-mode resonance filter array," Opt. Lett., vol. 36, no. 23, pp. 4698-4700, 2011.

[49] N. Ganesh, I. D. Block, and B. T. Cunningham, "Near ultravioletwavelength photonic-crystal biosensor with enhanced surface-to-bulk sensitivity ratio," Appl. Phys. Lett., vol. 89, p. 023901, 2006.

[50] J.-N. Liu, M. V. Schulemerich, R. Bhargava, and B. T. Cunningham, "Optimally designed narrowband guided-mode resonance reflectance filters for mid-infrared spectroscopy," Opt. Express, vol. 19, no. 24, pp. 24182-24197, 2011.

[51] Y. Kanamori, T. Kitani, and K. Hane, "Control of guided resonance in a photonic crystal slab using microelectromechanical actuators," Appl. Phys. Lett., vol. 90, p. 031911, 2007.

[52] F. Yang, G. Yen, and B. T. Cunningham, "Voltage-tuned resonant reflectance optical filter for visible wavelengths fabricated by nanoreplica molding," Appl. Phys. Lett., vol. 90, no. 26, p. 261109, 2007.

[53] N. Destouches, J.-C. Pommier, O. Parriaux, T. Clausnitzer, N. Lyndin, and S. Tonchev, "Narrow band resonant grating of 100\% reflection under normal incidence," Opt. Express, vol. 14, no. 26, pp. 12613-12622, 2006.

[54] A. B. Greenwell, S. Boonruang, and M. G. Moharam, "Multiple wavelength resonant grating filters at oblique incidence with broad angular acceptance," Opt. Express, vol. 15, no. 14, pp. 8626-8638, 2007.

[55] M. Shokooh-Saremi and R. Magnusson, "Multi-level periodic leaky-mode resonance elements: Design and applications," OSA Annual Meeting on Optical Interference Coatings (OIC 2010), Tucson, USA, WB2, June 2010.

[56] P. C. Mathias, N. Ganesh, W. Zhang, and B. T. Cunningham, "Graded wavelength one-dimensional photonic crystal reveals spectral characteristics of enhanced fluorescence," J. Appl. Phys., vol. 103, no. 9, p. 094320, 2008.

[57] Z. A. Roth, P. Srinivasan, M. K. Poutous, A. J. Pung, R. C. Rumpf, and E. G. Johnson, "Azimuthally varying guided mode resonance filters," Micromachines, vol. 3, pp. 180-193, 2012.

[58] Y. Ohtera, D. Kurniatan, and H. Yamada, "Design and fabrication of multi-channel $\mathrm{Si} / \mathrm{SiO}_{2}$ autocloned photonic crystal edge filters," Applied Optics, vol. 50, no. 9, pp. C50-C54, 2011.

[59] Y. Ohtera, K. Miura, and T. Kawashima, "Ge/SiO 2 photonic crystal multi-channel wavelength filters for short wave infrared wavelengths," Jpn. J. Appl. Phys., Part 1, vol. 46, no. 4A, pp. 1511-1515, 2007.

[60] Y. Ohtera, Y. Inoue, and T. Kawashima, "Sharp edge wavelength filters 
utilizing multilayer photonic crystals," Optics Express, vol. 17, no. 8, pp. 6347-6356, 2009.

[61] Y. Ohtera and T. Kawashima, "Extremely low optical transmittance in the stopbands of photonic crystals," Photonics and Nanostructures Fundamentals and Applications, vol. 7, no. 2, pp. 85-91, 2009.

[62] S. Yamaguchi, Y. Ohtera, and H. Yamada, "Four-channel spectral imaging in visible wavelength utilizing photonic crystal patterned wavelength filters," 17th Microoptics Conference (MOC'11), Sendai, Japan, B-3, Oct. 2011.

[63] Y. Ohtera, K. Daniel, and H. Yamada, "9-channel photonic crystal mosaic filters for near infra-red spectroscopic imaging," The 15th OptoElectronics and Communications Conference (OECC2010), Sapporo, Japan, 9E3-4, July 2010.

\section{Introduction}

Photonic crystal $(\mathrm{PhC})$ [1] is a class of dielectric material having refractive index modulation of the order of sub-wavelength scale. From the early years of $\mathrm{PhC}$ research, major trend has been to make use of photonic bandgap [2], such as the creation of waveguides and nanocavities.

On the other hand, another trend is emerging to utilize its diffraction and dispersion characteristics. In this paper we review recent progress of $\mathrm{PhC}$ 's application to spectrum analysis, in which the above effects play a key role.

This paper consists of five sections. In section 2, planar optical circuittype spectrometers and wavelength demultiplexers (DEMUX) will be introduced. All of the waveguide separation process is done in the waveguiding layer. We will call this "in-plane type" device for convenience.

In section 3, out-of-plane type devices will be explained. Grating couplers and $\mathrm{PhC}$ nanocavities are building blocks for this type. Signal light from a waveguide is directed out of the plane. In the following section, spectrometers utilizing guided-mode resonance (GMR) of sub-wavelength gratings (SWGs) and $\mathrm{PhCs}$ will be reviewed. Here, both input and output waves propagate normally to the plane of substrate. In the final section surface-normal type wavelength filters are dealt with. Both GMR type and Bragg reflection type filters will be described.

\section{In-plane type dispersion components/devices}

\subsection{Integrated spectrometer utilizing diffraction grating}

There is a large demand for small-size integrated spectrometers in the fields of biology and chemical sensing [3, 4], where online and field measurement are sometimes required. A trial to integrate spectrometer functions on a planar optical circuit has begun in 1990 in accordance with the progress of fabrication process. Typical device is the integrated spectrometer consisting of an etched diffraction grating (EDG) and a concave mirror on a slab waveguide. For example, Sanders et al. demonstrated sidewall gratings and 


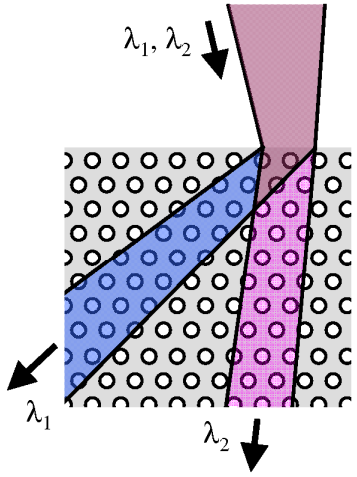

(a)

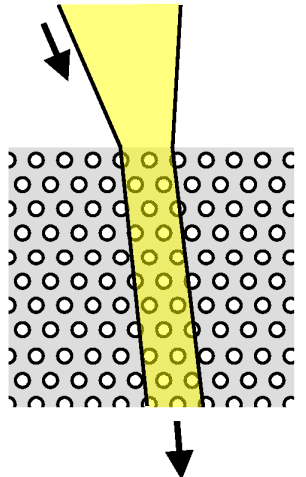

(b)

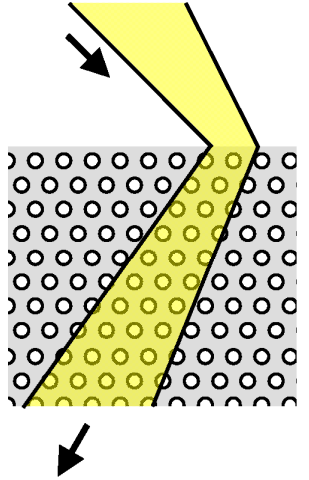

(c)

Fig. 1. Three kinds of abnormal refraction at $\mathrm{PhC}$ boundary. (a) superprism effect. (b) diffraction suppressed propagation. (c) negative refraction.

focusing function of wavelength-demultiplexed light on a SiON waveguide [5]. EDG type DEMUX was also fabricated in InGaAsP [6] and Si platforms [7].

Early application of $\mathrm{PhC}$ to the above EDG spectrometer was to use it as a part of concave mirror. Momeni and others embedded an array of holes (1-D PhC) inside the mirror section to increase the reflectivity [8].

\subsection{Superprism effect and negative refraction in photonic crystals}

Dispersion relation of $\mathrm{PhC}$ depends not only on constituent material but also on the ambient refractive index. A group of Agilent Co. Ltd. proposed a simple refractive index sensor based on this property. Their sensor consisted of a finite $\mathrm{PhC}$ slab and detected the index change of the cap layer (analyte) through the shift of transmission spectrum [9].

Device concept that makes full use of PhC's dispersion characteristics will be the one using superprism or negative refraction effects. Similar kind of abnormal refraction phenomena at the boundary of periodic waveguide has been theoretically predicted and experimentally demonstrated by Russell et al. [10] before the superprism was widely known. As the fabrication of multi-dimensional $\mathrm{PhC}$ has been made possible by the improvement of nano-fabrication technology, the above beam deflection phenomena was soon widely recognized among $\mathrm{PhC}$ community. The trigger of the following research was the experimental demonstration of superprism effect by Kosaka et al. [11, 12]. They observed highly wavelength sensitive refraction [11] on a multilayer type $\mathrm{PhC}$ fabricated by the autocloning method [13]. They also observed diffraction-free propagation and negative refraction [12]. Fig. 1 shows three representative effects of superprism.

Later, the boundary between $\mathrm{PhC}$ and input/output space was found to be critical for the shaping of output beams. To reduce unwanted crosstalk and the formation of stray light, $\mathrm{Wu}$ et al. made semicircular type $\mathrm{PhC}$ superprism [14].

Superprism phenomenon was explained as the result of the deformation 


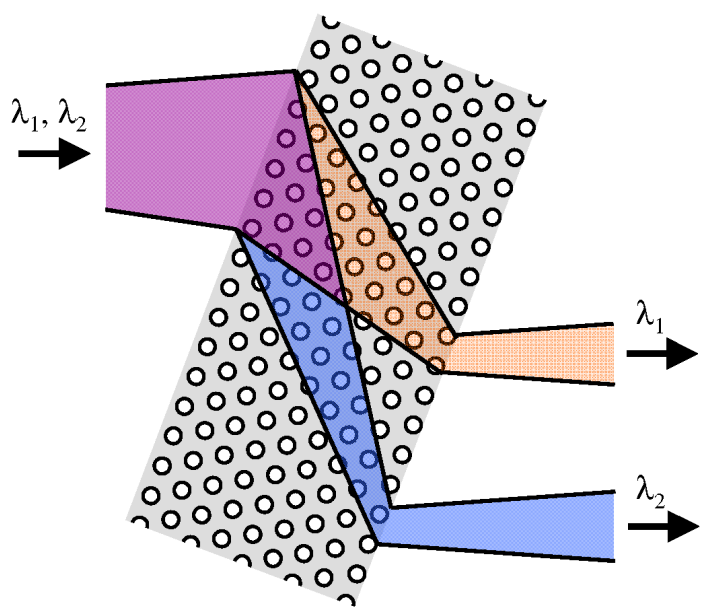

Fig. 2. Typical configuration for spectral analysis using superprism.

of equi-frequency surface (EFS) of dispersion relation. Near the photonic band gap the EFS shows partially negative curvature, leading to abnormal diffraction. If the EFS is highly deformed and becomes almost circular with negative curvature, negative refraction occurs at such frequency [15]. By properly incorporating negative refraction, the distortion and aberration due to superprism can be compensated. DEMUX having high beam quality and low insertion losses have been demonstrated on such composite prism layout $[16,17,18]$.

\subsection{Spectrometer and DEMUX utilizing superprism effect}

Fig. 2 shows a typical layout of superprism DEMUX. The signal light is expanded (if necessary) before incident on $\mathrm{PhC}$. The beam is deflected with wavelength dependent angle. $\mathrm{PhC}$ is designed so that the beam degradation by abberation becomes minimum.

Bakhtazad et al. demonstrated spectrum separation using the most fundamental geometry: triangular superprism made of 1-D PhC [19]. Matsumoto et al. demonstrated DEMUX for WDM by combining superprism and superlens [20]. By optimizing lattice geometry and anti-reflective structure, they realized low insertion loss and low aberration. Combination of S-vector and k-vector superprisms were also proposed [21]. Bernier reported 4-ch DEMUX for $1.55 \mu \mathrm{m}$ CWDM using rhombohedral type large area $\mathrm{PhC}$ [22]. Visible spectrometer (center $\lambda \sim 650 \mathrm{~nm}, 7 \mathrm{ch}$, wavelength spacing $\sim 1 \mathrm{~nm}$ ) has been demonstrated using SiN [23]. In Ref. [24] beam diffraction was compensated by cascading a pair of triangular superprisms.

All of the above devices were realized in 2-D $\mathrm{PhC}$ slab waveguides. On the other hand, Kim et al. demonstrated 20-ch spectrometer component using $3 \mathrm{D}-\mathrm{PhC}$ in the form of free-standing plastic plate. Their $\mathrm{PhC}$ was colloidal silica in polymer [25]. 


\section{Out-of-plane type spectrometer}

\subsection{Grating coupler-type devices}

Periodic waveguides support leaky modes for wavelengths shorter than its period. This mode radiates power out of the waveguide. The angle of radiation is a function of the pitch and wavelength. This kind of waveguide has been called grating coupler (GC) and utilized to guide external wave into the slab and vice versa [26]. GC is still regarded as a key component in modern optics: in the silicon photonics field, it is used to connect external fibers and waveguide, and to transmit signal between waveguides in different layers [27, 28]. A number of design theories have been reported. Especially, spatial profile of radiated beam can now be tailored $[29,30]$. This is one of the important technology for out-of-plane type spectrometers.

Fundamental concept of GC as a sensor is to detect the change of refractive index above the grating region through the shift of loss or radiation spectra [31, 32]. Out-of-plane type $\mathrm{PhC}$ spectrometers and sensors are based on this principle. The group at Columbia University demonstrated 9-ch spectrometer at visible wavelength [33]. They created 2-D PhC GC with different lattice constant and extracted signal light of different wavelengths as shown in Fig. 3. As the number of channels is small compared to EDG-type spectrometer, direct acquisition of spectrum is usually difficult: instead, they estimated the spectrum by considering both spectral response of each $\mathrm{PhC}$ GC and measured channel intensities.

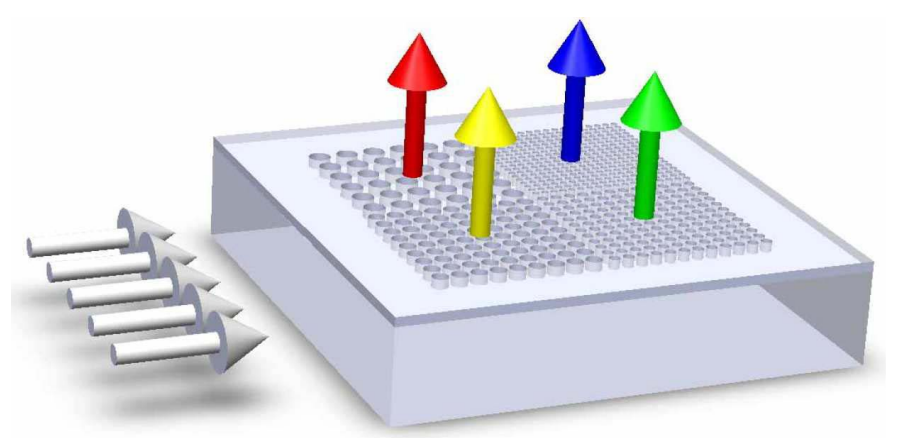

Fig. 3. Schematic of spatially resolved, wavelengthselective outcoupling of waveguided light using an array of PhCs [33]. Copyright: 2010 Optical Society of America.

\subsection{Spectrometer utilizing photonic crystal nanocavities}

Nanocavities created in 2-D PhC slab can also be used to emit light with a specified wavelength. The relationship between the geometry of the nanocavity and the resonance mode, radiation pattern was systematically analyzed by a number of groups [34]. A 100ch spectrometer for a wavelength range of $804 \mathrm{~nm} \sim 840 \mathrm{~nm}$ (spacing: $0.35 \mathrm{~nm}$ ) was demonstrated by a group in Columbia university [35]. They formed L3 cavity of $\mathrm{Q}=2000 \sim 3000$ in GaP PhC slab, and observed sharp radiation spectrum from each cavity region. 


\section{Spectrum measurement devices utilizing Guided-mode res- onance}

\subsection{Guided-mode resonance}

When a sub-wavelength grating (SWG) is illuminated by a plane wave, the reflectivity of 0th order wave reaches as high as $100 \%$ at a specific wavelength. This phenomenon has been called guided-mode resonance (GMR) and utilized as a key mechanism for sharp wavelength selective filters [36]. GMR is nothing but the reverse process of GC; i.e., plane wave excites leaky modes, and diffracted back to the incident space at a resonant wavelength [37]. It was also shown that GMR also occurs in PhC slab [38]. The narrow-band, $100 \%$ reflectivity has been experimentally demonstrated [39].

Recent improvements of semiconductor manufacturing process made it possible to fabricate SWGs made of high index materials such as Si. These materials have extremely large index contrast with substrate (typically $\Delta \mathrm{n} \sim 2.0$ ). Therefore they are sometimes called HCG (High Contrast Grating) [40]. Reflection spectrum of HCG is highly modificated from that of slab structure. This property is used to design a broadband reflection mirror, which was difficult in conventional low index contrast SWGs. Almost all the spectrometers described in the following section employ HCG or $\mathrm{PhC}$.

\subsection{Sensor application of GMR}

Several groups demonstrated biosensors that detect the adhesion of protein molecule on the SWG or $\mathrm{PhC}$, through the change of resonance wavelength of reflection by GMR. Schmid at NRC reported large area $\mathrm{PhC}$ sensor region using SOI wafer [41]. Block et al. fabricated $\mathrm{TiO}_{2}$ periodic structures on an epoxy [42] and porous $\mathrm{SiO}_{2}$ undercladding [43] and reported the sensitivity of $\Delta \lambda / \Delta n=306.7 \mathrm{~nm} / \mathrm{RIU}$ (refractive index unit).

Recently Cunningham et al. in Univ. Illinois established fabrication process of multichannel $\mathrm{PhC}$ sensor plate on a plastic well which is commonly used in chemical/biological experiments. Typical reflection spectrum from each $\mathrm{PhC}$ region [45] and the picture of the plates [44] are shown in Fig. 4. In their system the analyte can be directly put on the plate. Reflection characteristics of each well were measured by scanning an array of fiber probes $[45,46]$.

\subsection{Sub-wavelength grating (SWG) filters}

In this section some of the wavelength filters made of SWG and $\mathrm{PhC}$ are reviewed. As shown in the previous part major advantage of such microstructured filters is the parallel integration capability. By fabricating a number of microfilters with different spectral characteristics on a common substrate, it can be directly applied to a spectral sensing device.

The simplest function is the coarse separation of broadband signal. In Ref. [47] and [48] RGB color separation using GMR filters were reported. The wavelength range of operation can be extended from conventional visible/NIR 


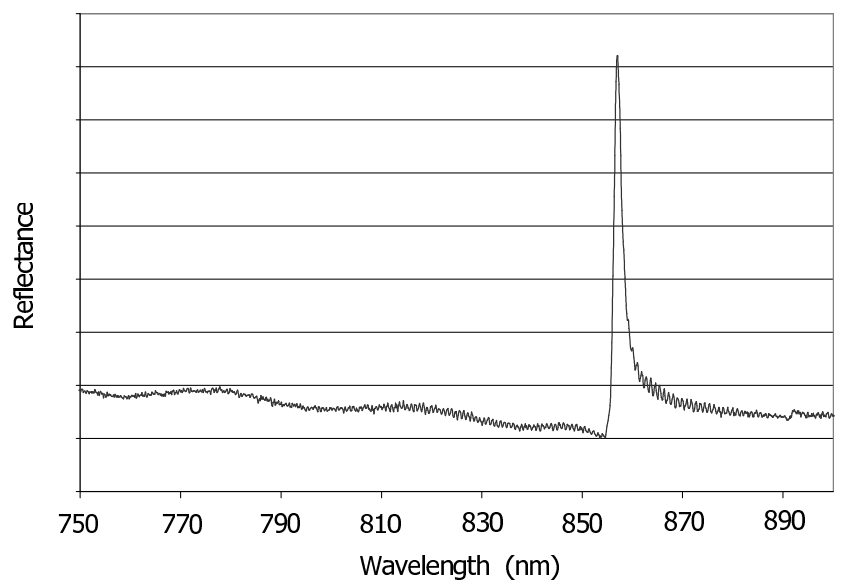

(a)

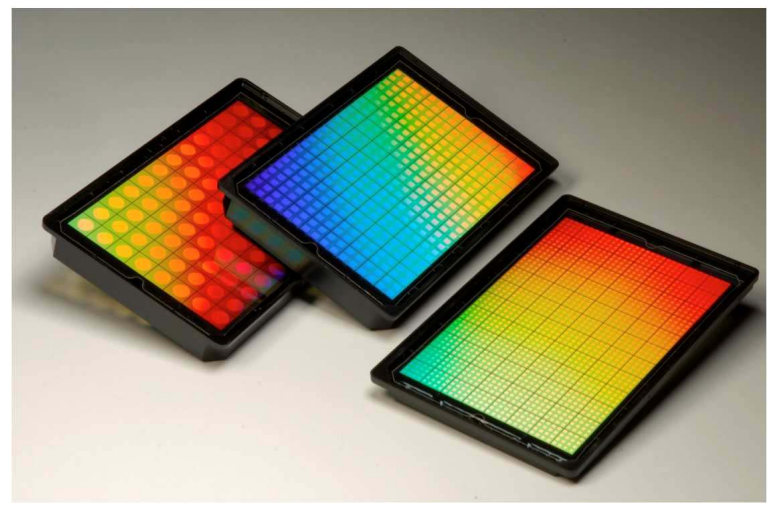

(b)

Fig. 4. Multichannel PhC biosensors. (a) Typical reflection spectrum of $\mathrm{PhC}$ part, (b) Picture of $\mathrm{PhC}$ biosensors on 96, 384, and 1536-well microplates. See [45] and [44] for detail. (figures by courtesy of Prof. B. T. Cunningham)

to UV [49] and mid-IR [50] by an appropriate choise of materials and grating design.

Tunability of the reflection wavelength is another important functions from a practical viewpoint. Kanamori et al. fabricated MEMS-driven SWG filters using SOI substrate. Changing the resonance wavelength was achieved by the control of applied voltage [51]. Usage of liquid crystal overclad layers was also found effective to get tunability [52].

Conventionally, a single high index film has been used for SWG. On the other hand, the feasibility of multilayer or multilevel structures were investigated to improve filter performances $[53,54,55]$. SWGs with spatially graded structure have also been studied to obtain contiguous reflection spectrum $[56,57]$.

\section{Multilayer photonic crystal filters (ML-PhCF) and its appli- cation to spectral imaging}

In this section another type of $\mathrm{PhC}$ filters that mainly make use of Bragg reflection are introduced. We have been investigating the fabrication process 


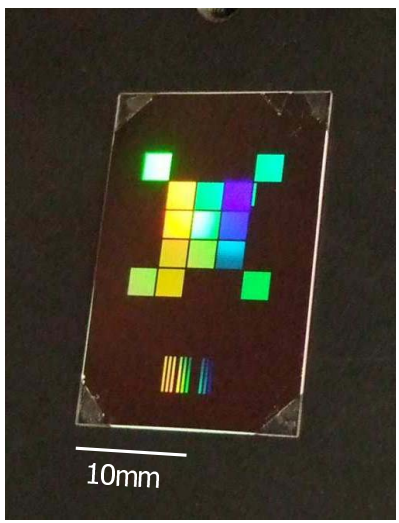

(a)

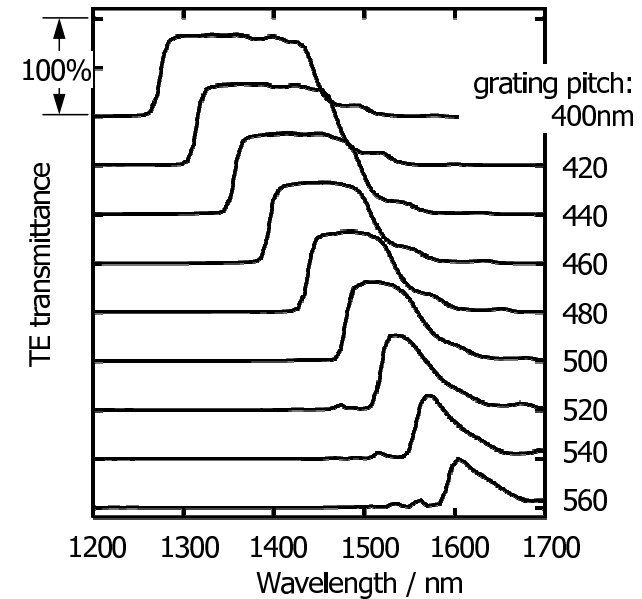

(b)

Fig. 5. Multilayer type $\mathrm{PhC}$ filter. (a) Exterior view. Each square region has different grating pitch and therefore passes different wavelength band. (b) measuret transmission spectra.

for multilayer on grating, and exploring its filter functions. The advantage of using such a structure is its broadband reflection/transmission characteristics and parallel integration capability. To date, multichannel filters for visible, near infrared [58] and short wave infrared [59] were demonstrated.

Fig. 5 shows an example of $\mathrm{Si} / \mathrm{SiO}_{2}$ multilayer $\mathrm{PhC}$ filters for NIR range. We fabricated this filter using bias sputtering based process (autocloning method [13]). By balancing the effect of simple deposition and sputter etching, stable wavy interface was automatically replicated. Each square region of $2.4 \times 2.4 \mathrm{~mm}$ functions as a bandpass filter. Transmission band linearly shifted corresponding to the grating pitch. Total shift of the center wavelength was measured as $>250 \mathrm{~nm}$.

We found that this kind of multilayer exhibited extremely sharp cutoff characteristics [60] when it is used as an edge filter. Origin of this characteristics was explained by the coupling between plane-wave like modes that proagate normally to the layers, and obliquely propagating mode that is diffracted by the grating structure [61].

We also fabricated 4-ch Bayer pattern filters for visible wavelength using $\mathrm{Nb}_{2} \mathrm{O}_{5} / \mathrm{SiO}_{2}$. The filter was directly mounted on a CCD image sensor. Pictures of the PhC-integrated CCD and the camera are shown in Fig. 6. Main application of this camera system is the non-invasive monitoring of hemoglobin concentration for biomedical targets. Therefore the transmission bands of the filters were optimized to detect the absorption bands of hemoglobin. In Fig. 6 test targets ((c), blood sample dispersed in a gelatin with graded thickness) and the result of measurement (d). The images through PhC filters were passed to de-mosaicing process, and four pairs of images corresponding to each wavelength band was created. From these images we reconstructed spatial distribution of the total amount of hemoglobin. In this process we employed principal component analysis (PCA) [62]. As 
PhC 4ch bayer pattern filter

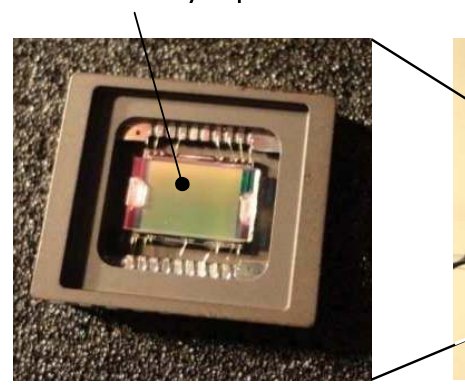

(a)

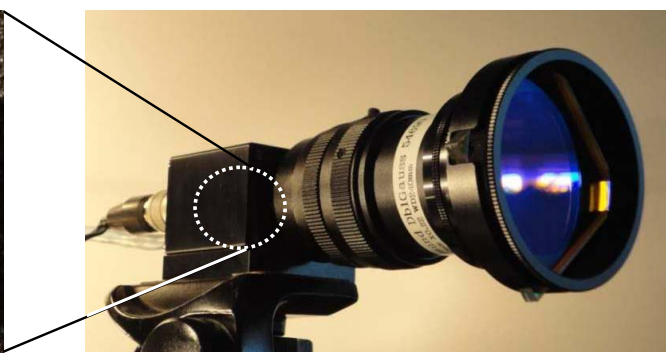

(b)
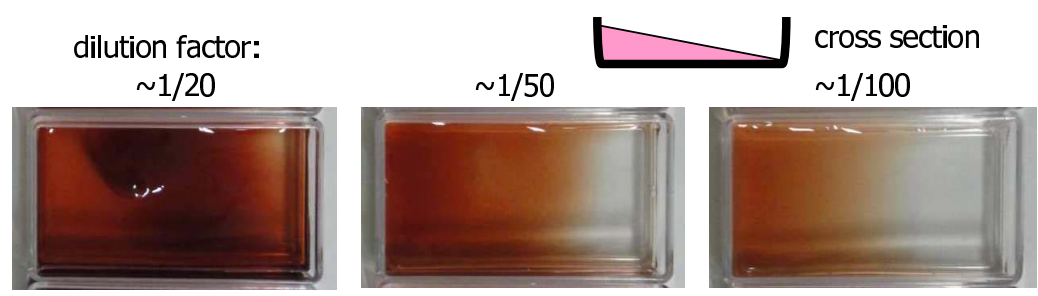

(c)
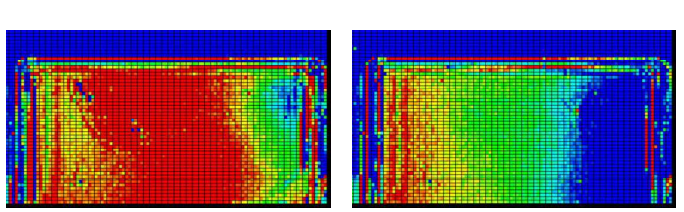

(d)

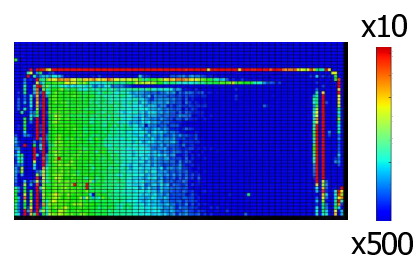

Fig. 6. Multilayer PhC filters and its application to spectral imaging. (a) Picture of an image sensor. $4 \mathrm{ch} \mathrm{PhC}$ filter was mounted on CCD. (b) exterior view of the CCD camera. (c) hemoglobindispersed gelatin sample with various thickness. (d) hemoglobin concentration map reconstructed from 4ch spectral images.

can be seen, dynamic range of the total hemoglobin contents (dillution ratio to the reference blood sample) was found to be quite high.

This kind of PCA-assisted imaging was also appricable to NIR. We fabricated $\mathrm{Si} / \mathrm{SiO}_{2}$ 9ch $\mathrm{PhC}$ multilayer filters and carried out spectral imaging of liquid samples. Measurement of the mixture ratio of water and ethanol were successfully achieved with good accuracy [63].

\section{Conclusion}

The application of sub-wavelength gratings and photonic crystals to spectrometers and wavelength filters were reviewed. Three types of spectrometers were described: (1) in-plane type, which mainly utilizes superprism effect of PhC. (2) out-of-plane type, which uses grating coupler or photonic crystal nanocavities. (3) full surface-normal reflection type sensors. In the last part we introduced several examples of SWGs and multilayer PhCs as spectral filtering components. 

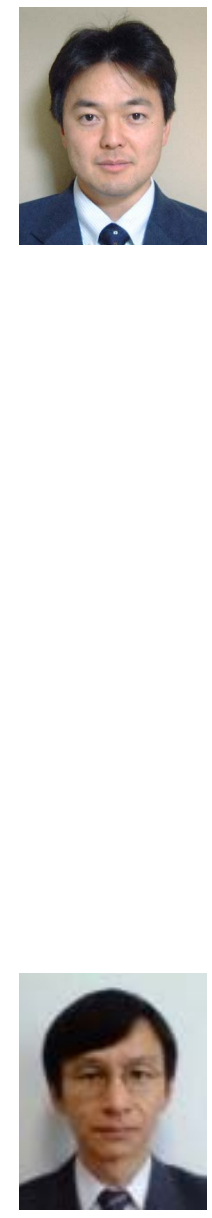

\section{Yasuo Ohtera}

received B.E., M.E., and Ph.D. degrees in electronic engineering from Tohoku University, Sendai, Japan, in 1992, 1994, and 1997, respectively. In 1997, he joined the Research Institute of Electrical Communication, Tohoku University, as a research associate. In 2001 he became a research fellow of the New Industry Creation Hatchery Center (NICHe), Tohoku University. From 2004 he spent four years as an associate professor in Biomedical Engineering Research Organization of Tohoku University (TUBERO). In 2008 he joined Graduate School of Engineering, T.U. His current research interest is the development of micro-photonic devices for sensing and communications. Dr. Ohtera is a member of the Institute of Electronics, Information and Communication Engineers (IEICE) of Japan, the Japan Society of Applied Physics (JSAP), and the Optical Society of America (OSA).

\section{Hirohito Yamada}

received the B.E. degree from Kanazawa University in 1981, and the M.E. and Ph.D. degrees from Tohoku University in 1983 and 1987 respectively, all in electronics engineering. In 1987 he joined the Opto-Electronics Research Laboratories, NEC Corporation, where he studied laser diodes for optical communications. From 1991 to 1997 he developed laser diodes for optical-subscriber systems in Kansai Electronics Research Laboratory. From 1998, he studied photonic crystals and Si nano-wire devices in NEC Tsukuba. Since 2006, he has been a Professor at the Department of Electrical and Communication Engineering, Tohoku University, Sendai, Japan. Dr. Yamada is a member of the Institute of Electronics, Information and Communication Engineers (IEICE), the Institute of Electrical and Electronics Engineers (IEEE), the Japan Society of Applied Physics (JSAP), and the Optical Society of America (OSA). 